\title{
Article
}

http://dx.doi.org/10.11646/phytotaxa.172.3.6

\section{Silene peloritana (Caryophyllaceae) a new species from Sicily}

\author{
CRISTIAN BRULLO, SALVATORE BRULLO*, GIANPIETRO GIUSSO DEL GALDO, PIETRO MINISSALE \& \\ SAVERIO SCIANDRELLO \\ Dipartimento di Scienze Biologiche, Geologiche e Ambientali, Sez. Biologia Vegetale, Università di Catania, via A. Longo, 19, I - 95125 \\ Catania (Italy); e-mail: salvo.brullo@gmail.com \\ *Author for correspondence
}

\begin{abstract}
Silene peloritana is described and illustrated as a narrow endemic circumscribed to Mt. Scuderi, an isolated peak of the Peloritani range (NE Sicily). The new species, belonging to $S$. sect. Dipterosperma, is a small therophyte growing on cacuminal carbonatic stands. It is well differentiated from the other Sicilian species of this section for its small size, shorter lower internodes, bracts and calyx teeth, petal lobes obovate, coronal scales purplish, and anthers pinkish-violet. A key of the Sicilian taxa belonging to Silene sect. Dipterosperma is provided.
\end{abstract}

Key words: Caryophyllaceae, chorology, ecology, Flora of Sicily, Silene, taxonomy

\section{Introduction}

Silene Linnaeus (1753: 416) sect. Dipterosperma (Rohrbach 1868: 144.) Chowdhuri (1957: 248) includes a small group of annual species with stem hairy-pubescent, eglandular, flowers erect, arranged in monochasia or dichasia, calyx hairy, not inflated, with 10-nerved, anthophore pubescent, petal limb deeply bifid, coronal scales present, seeds orbicular-reniform, laterally flat and dorsally furrowed between two undulate wings (Maire 1963, Talavera 1990, Chater et al. 1993). This group has been recently surveyed by Valsecchi (1995) and Brullo et al. (2012) who highlighted the remarkable variability among the populations, most of which require further studies.

During the field investigations carried out in the central Mediterranean territories, an interesting population belonging to this section was found on the top of Mt. Scuderi, an isolated peak of the massif Peloritani (NE Sicily). The population found differs from the current known species of the sect. Dipterosperma, which occur in the Mediterranean and Irano-Turanian countries, by several morphological features chiefly regarding habit, leaves, inflorescences, flowers, capsules and seeds. Moreover, there are both geographic and ecologic separations from the other species of Silene sect. Dipterosperma currently recorded from Sicily [such as $S$. colorata Poiret (1789:163), widespread along the coastal areas of Sicily, S. apetala Willdenow (1799: 703) known only for the island of Linosa (Pelagian Archipelago), S. nummica Valsecchi (1995: 452) and S. arghireica Valsecchi (1995: 459), both circumscribed to few localities of the Sicilian western coasts, and lastly S. kemoniana C.Brullo, Brullo, Giusso, Ilardi \& Sciandr. (2012: 209) recently described as a punctiform endemism located in a mountain area close to Palermo]. As new species for science (named Silene peloritana, see below) is here proposed and described.

\section{Material and methods}

The morphological study was carried out on living plants (20 specimens), collected from the locus classicus. The morphological comparison with the related species of $S$. sect. Dipterosperma was made using herbarium material preserved in CAT, literature data, and living plants cultivated at the Botanical Garden of Catania University. The micro-morphology of the seed testa has been studied on dried material with the aid of a Scanning Electron Microscope (SEM) Zeiss EVO LS10, according to the Huttunen \& Laine (1983) protocol. 
TABLE 1. (Continued)

\begin{tabular}{|c|c|c|c|}
\hline & S. arghireica & S. kemoniana & S. apetala \\
\hline Plant height (cm) & $20-35$ & $5-15(-20)$ & $10-40$ \\
\hline Plant indumentum & minutely pubescent & pilose-pubescent & densely pubescent \\
\hline Hair length (mm) & up to 1 & up to 1 & up to 1 \\
\hline Stem & erect & prostrate-ascending & erect \\
\hline Stem branching & at the base and nodes & at the base & no o few branched \\
\hline Lower internodes length $(\mathrm{cm})$ & $1-3$ & $1-2$ & $1-2$ \\
\hline Upper internodes length $(\mathrm{cm})$ & up to 5 & up to 4 & up to 5 \\
\hline Leaf shape & lanceolate, thin & spathulate, thin & lanceolate to linear-lanceolate \\
\hline Leaf size (mm) & $10-35 \times 4-8$ & $8-20 \times 2-6$ & $10-20 \times 2-8$ \\
\hline Leaf nerves & 1-nerved & 1-nerved & 1-3-nerved \\
\hline Leaf apex & sub-acute & obtuse & apiculate \\
\hline Inflorescence (shape) & dichasia & helicoid monochasia & irregular monochasia-dichasia \\
\hline Inflorescence (no. flowers) & $3-5$ & $(1-) 2-4(-5)$ & $1-4$ \\
\hline Bract length $(\mathrm{mm})$ & $3-10$ & $4-10$ & $5-10$ \\
\hline Pedicel length (mm) & $4-15$ & $3-15$ & $5-20$ \\
\hline Calyx indumentum & minutely pubescent & minutely pubescent & scabrid-hirsute \\
\hline Calyx length (mm) & $13-14$ & $11-13$ & $6-9$ \\
\hline Calyx teeth size (mm) & $3.8-4.5 \times 1.6-2.2$ & $1.8-2.2 \times 1.2-1.5$ & $2.5-3 \times 1.5-2$ \\
\hline Calyx teeth shape & triangular, acute & oblong, rounded & triangular-acuminate \\
\hline Corolla colour & pink & pink & pinkish \\
\hline Petal length (mm) & $18-20$ & $15-16$ & $0-9$ \\
\hline Petal limb length (mm) & $8-9$ & $8-8.5$ & $0-3$ \\
\hline Petal lobes size $(\mathrm{mm})$ & $3-3.2 \times 3-3.5$ & $6-6.5 \times 1.5-2$ & inconspicuous \\
\hline Petal lobes shape & hemi-elliptical & linear-spathulate & linear \\
\hline Petal lobes margin & smooth & smooth & smooth \\
\hline Petal claw length (mm) & $10-12$ & $7-8$ & $0-7$ \\
\hline Claw dorsal midrib & glabrous & glabrous & minutely hispid \\
\hline Coronal scales length (mm) & $2.5-3$ & $2.5-3$ & $0.8 \mathrm{~mm}$ \\
\hline Coronal scales shape & 2-lobed, white, smooth & $\begin{array}{l}\text { retuse, white, erose-incised } \\
\text { above }\end{array}$ & retuse, white, smooth \\
\hline Stamen filaments length (mm) & $9-10$ & $9-10$ & $5-8$ \\
\hline Anther & pink & pink & pale pink \\
\hline Anther length (mm) & $1.8-2$ & $1.6-1.8$ & 0.6 \\
\hline Ovary length (mm) & $3.5-4$ & $2.4-2.6$ & $2-3$ \\
\hline Capsula length (mm) & $7-8$ & $8-10$ & $4-6$ \\
\hline Carpophore & $7-8$ & $4-5$ & $0.5-1.5(2.5)$ \\
\hline Seed diameter $(\mathrm{mm})$ & $1.5-2$ & $1.6-1.7$ & $0.9-1.2$ \\
\hline
\end{tabular}

\section{Acknowledgements}

The authors wish to thank G. Fichera (University of Catania) for his helpful assistance with the Scanning Electron Microscopy.

\section{References}

Attar, F., Keshvari, A., Ghahreman, A., Zarre, S. \& Aghabeigi, F. (2007) Micromorphological studies on Verbascum (Scrophulariaceae) in Iran with emphasis on seed surface, capsule ornamentation and trichomes. Flora 202: 169-175.

Bartolo, G., Brullo, S. \& Pulvirenti, S. (1994) Su una nuova associazione della classe Cytisetea striato scoparii in Sicilia. Bollettino Accademia Gioenia di Scienze Naturali Catania 27(346): 390-407.

Becker, W. (1905) Die systematische Behandlung der Formenkreise der Viola calcarata und lutea (im weitesten Sinne genommen) auf Grundlage ihrer Entwicklungsgeschichte. Beihefte zum Botanischen Centralblatt 18: 347-393.

Berggren, G. (1981) Atlas of seeds and small fruits of Northwest-European plant species with morphological descriptions. Part 3, Salicaceae-Cruciferae. Swedish Museum of Natural History, Stockholm, 261 pp. 
Brullo, C. \& Brullo, S. (2009) Considerazioni su alcune specie critiche della flora sicula. In: Peccenini, S. \& Domina, G. (eds.), Gruppi Critici della Flora d'Italia. Luxograf s.r.1., Palermo, pp. 43-44.

Brullo, S. \& Guarino, R. (2001) Festuca humifusa (Gramineae), a new species from Sicily. Bocconea 13: 409-412.

Brullo, S., Minissale, P. \& Spampinato G. (1995) Considerazioni fitogeografiche sulla flora della Sicilia. Ecologia Mediterranea 21: 99-117.

Brullo S., Marcenò C. \& Siracusa G. (2004) La classe Asplenietea trichomanis in Sicilia. Colloques Phytosocioloiques 28: $467-538$.

Brullo, S., Guglielmo, G., Pasta, S., Pavone, P. \& Salmeri, C. (2008) - Note citotassonomiche sul genere Thapsia (Apiaceae). Informatore Botanico Italiano 40 (Suppl. 1): 41-48.

Brullo, C., Brullo, S., Giusso del Galdo, G. \& Scuderi, L. (2009) Taxonomical notes on the Sicilian populations of Asperula gussonei (Rubiaceae): A. peloritana sp. nov. Anales del Jardin Botanico de Madrid 66: 85-92. http://dx.doi.org/10.3989/ajbm.2180

Brullo, C., Minissale, P., Sciandrello, S. \& Spampinato, G. (2011) Evaluation of the endemic vascular flora of Hyblaean territory (SE Sicily-Italy). Acta Botanica Gallica 158: 617-631. http://dx.doi.org/10.1080/12538078.2011.10516299

Brullo, C., Brullo, S., Giusso del Galdo, G., Ilardi,V. \& Sciandrello, S. (2012) Anew species of Silene sect. Dipterosperma (Caryophyllaceae) from Sicily. Anales del Jardin Botanico de Madrid 69: 209-216. http://dx.doi.org/10.3989/ajbm.2327

Chater, A.O., Walters, S.M. \& Akeroid, J.R. (1993) Silene L. In: Tutin, T.G., Burges, N.A., Chater, A.O., Edmodson, J.R., Heywood, V.H., Moore, D.M., Valentine, D.H., Walters, S.M. \& Webb, D.A. (eds.), Flora Europaea, ed. 2, 1. Cambridge University Press, Cambridge, pp. 191-218.

Chowdhuri, P.K. (1957) Studies in the genus Silene. Notes from the Royal Botanic Garden, Edinburgh 22: 221-278.

Di Gristina, E., Gottschlich, G., Galesi, R., Raimondo, F.M. \& Cristaudo, A. (2013) Hieracium hypochoeroides subsp. montis-scuderii (Asteraceae), a new endemic subspecies from Sicily (Italy). Flora Mediterranea 23: 49-55. http://dx.doi.org/10.7320/flmedit23.049

El-Oqlah, A.A. \& Karim, F.M. (1990) Morphological and anatomical studies of seed coat in Silene species (Caryophyllaceae) from Jordan. Arab Gulf Journal of Scientific Research 8: 121-139.

Fawzi, N.M., Fawzy, A.M. \& Mohamed, A.A-H. (2010) Seed morphological studies on some species of Silene L. (Caryophyllaceae). International Journal of Botany 6: 287-292.

http://dx.doi.org/10.3923/ijb.2010.287.292

Gibson, S. (1843) Notes on an apparently undescribed Hieracium. Phytologist 1: 741-742.

Gussone, G. (1827) Florae Siculae Prodromus, Addenda et Emendandam 1. Regia Typographia, Neapoli, 11 pp.

Gussone, G. (1828) Florae Siculae Prodromus 2. Regia Typographia, Neapoli, 585 pp.

Hong, S.P., Han, M.J. \& Kim, K.J. (1999) Systematic significance of seed coat morphology in Silene L. s.str. (Sileneae-Caryophyllaceae) from Korea. Journal of Plant Biology 42: 146-150. http://dx.doi.org/10.1007/bf03031023

Hosny, A. \& Zareh, M. (1993) Taxonomic studies of Silenoideae (Caryophyllaceae) in Egypt. 2. Taxonomic significance of seed characters in the genus Silene L. with special reference to the seed sculpture. Tackholmia 14: 37-44.

Huttunnen, S. \& Laine, K. (1983) Effects of air-born pollutants on the surface wax structure of Pinus sylvestris needles. Annales Botanici Fennici 20: 79-86.

IUCN (2010) The IUCN red list of threatened species, version 2010.4. IUCN Red List Unit, Cambridge, U.K. Available from: http://www. iucnredlist.org/ (accessed 20 January 2014).

Johnson, L.A., Huish, K.H. \& Porter, J.M. (2004) Seed surface sculpturing and its systematic significance in Gilia (Polemoniace) and segregated genera. International Journal of Plant Sciences 165: 153-172.

http://dx.doi.org/10.1086/380748

Kerner, A. (1874) Floristische Notizien. Oesterreichische Botanische Zeitschrift 24: 169-172. http://dx.doi.org/10.1007/bf01625971

Lacaita, C. (1915) Piante italiane critiche e rare. Nuovo Giornale Botanico Italiano, nuova serie, 22: 236-248.

Linnaeus, C. (1753) Species Plantarum 1-2. Laurentii Salvii, Holmiae, 1200 pp

Linnaeus, C. (1762) Species Plantarum, ed. 2, 1. Laurentii Salvii, Holmiae, 784 pp.

Linnaeus, C. (1767) Mantissa Plantarum. Laurentii Salvii, Holmiae, 588 pp.

Lojacono Pojero, M. (1907) Flora Sicula 2(2). Tipo-Litografia Salvatore Bizzarrilli, Palermo, 428 pp.

Maire, R. (1963) Flore de l'Afrique du Nord, Vol. 10. Dicotyledonae: Centrospermales: Caryophyllaceae, sf. Silenoideae. Encyclopédie Biologique 62: 1-336.

Moazzeni, H., Zarres, S., Al-Shehbaz, I.A. \& Mummenhoff, K. (2007) Seed-coat microsculpturing and its systematic application in Isatis 
(Brassicaceae) and allied genera in Iran. Flora 202: 447-454.

http://dx.doi.org/10.1016/j.flora.2006.10.004

Moench, C. (1794) Methodus Plantas Horti Botanici et Agri Marburgensis. Officina Nova Libraria Accademiae, Marburgi Cattorum, 800

pp.

http://dx.doi.org/10.5962/bhl.title.304

Ocaña, M.A., Juan, R., Fernàndez, I \& Pastor, J. (2011) Estudio morfológico de semillas de Silene (Caryophyllaceae) del Suroeste de España. Lagascalia 31: 21-45.

Peruzzi, L. \& Carta A. (2013) A taxonomic revision of Silene nocturna species complex (Caryophyllaceae) in Italy. Phytotaxa 88: 38-48

Peruzzi, L. \& Passalacqua N.G. (2008) Taxonomy of Onosma echioides (L.) L. complex (Boraginaceae) based on morphometric analysis. Botanical Journal of the Linnean Society 157: 768-774. http://dx.doi.org/10.1111/j.1095-8339.2008.00827.x

Perveen, A. (2009) Seed morphology of the genus Silene: Caryophyllaceae from Pakistan and Kashmir. International Journal of Biology and Biotechnology 6: 219-217.

Pignatti, S. (1982) Flora d'Italia 1. Edagricole, Bologna, 790 pp.

Poiret, J.L.M. (1789) Voyage en Barbarie 2. J.B.F. Née de la Rochelle, Paris, 319 pp.

Presl, C.B. (1822) Plantarum Rariorum Siciliae. In: Presl, J.S. \& Presl, C.B (eds.), Deliciae Pragenses. Calve, Prague, pp. 1-152.

Presl, C.B. (1826) Flora Sicula 1. A. Borrosch, Pragae, 216 pp.

Rohrbach, P. (1868) Monographie der Gattung Silene. Wilhelm Engelmann, Leipzig, 249 pp. http://dx.doi.org/10.5962/bhl.title.15462

Sciandrello, S., Brullo, C., Brullo, S., Giusso del Galdo, G., Minissale, P., Salmeri, C., (2013) A new species of Brassica sect. Brassica (Brassicaceae) from Sicily. Plant Biosystems 147: 812-820. http://dx.doi.org/10.1080/11263504.2013.829885

Sciandrello, S., Guarino, R., Minissale, P. Spampinato, G. (2014) The endemic vascular flora of Peloritani Mountains (NE Sicily): plant functional traits and phytogeographical relationships in the most isolated and fragmentary micro-plate of the Alpine orogeny. Plant Biosystems 148 (online). http://dx.doi.org/10.1080/11263504.2014.908978

Strobl, G. (1883) Flora der Nebroden. Flora 66: 547-554.

Talavera, S. (1990) Silene L. In: Castroviejo, S., Laìnz, M., Lòpez Gonzàlez, G., Montserrat, P., Muñoz Garmendia, F., Paiva, J. \& Villar, L. (eds.), Flora Iberica 2. Real Jardín Botánico C.S.I.C., Madrid, pp. 313-406.

Valsecchi, F. (1995) Indagini sistematiche, tassonomiche e corologiche nel gruppo Silene colorata Poir. - S. sericea All. - S. canescens Ten. Bollettino Società Sarda di Scienze Naturali 30: 447-476.

Villa, R. (1995) Diversità morfologica nei semi di alcune Silene del gruppo Silene colorata Poir. - Silene sericea Ten. Bollettino Società Sarda di Scienze Naturali 30: 477-488.

Villars, M. (1779) Prospectus de l'histoire des Plantes du Dauphiné. Imprimerie Royal, Grenoble, 57 pp.

Willdenow, C.L. (1799) Species Plantarum, ed. 4, 2(1). G.C. Nauk, Berolini, 823 pp.

Yildiz, K. (2006) A morphological investigation on Silene L., (Caryophyllaceae) species distributed in West Anatolia and North Cyprus. Pakistan Journal of Botany 38: 67-83.

Yildiz, K., \& Cirpici, A. (1998) Seed morphological studies of Silene L. from Turkey. Pakistan Journal of Botany 30: $173-188$.

Yildiz, K., \& Minareci, E. (2008) Morphological, anatomical, palynological and cytological investigation on Silene urvillei Scott. (Caryophyllaceae). Journal of Applied Environmental and Biological Sciences 2: 41-46.

Zareh, M. M. (2005) Seed diversity among certain species of Caryophyllaceae in Egypt. Pakistan Journal of Biological Sciences 8: $714-720$.

http://dx.doi.org/10.3923/pjbs.2005.714.720 